\title{
Electrical conditioning of adipose-derived stem cells in a multi-chamber culture platform
}

\author{
Pavesi $A^{\mathrm{a}}{ }^{.}$., Soncini $M^{\mathrm{a}}$., Zamperone $A^{\mathrm{c}}$., Pietronave $S^{\mathrm{c}} .$, Medico $E^{\mathrm{de}}$., Redaelli A. $^{a}$, \\ Prat $M^{\mathrm{cf}}$. and Fiore G. $B^{\mathrm{a}}$.
}

${ }^{a}$ Dipartimento di Elettronica, Informazione e Bioingegneria, Politecnico di Milano, Piazza Leonardo da Vinci 32, 20133, Milano, Italy

'Department of Health Sciences, Università del Piemonte Orientale “A. Avogadro", Via Solaroli 17, 28100, Novara, Italy

${ }^{\mathrm{d} I n s t i t u t e}$ for Cancer Research @ Candiolo, Str. Prov. 142, 10060, Candiolo (TO), Italy

${ }^{\mathrm{e}}$ Dipartimento di Oncologia, Università di Torino, Regione Gonzole 10, 10043, Orbassano (TO), Italy

${ }^{\mathrm{f}}$ Centro di Biotecnologie per la Ricerca Medica Applicata (BRMA) Via Solaroli 17, 28100 Novara, Italy

*Corresponding author

Andrea Pavesi, Ph.D.

Singapore MIT Alliance for Research and Technology, BioSystems and Micromechanics, 1 CREATE Way, \#04-13/14 Enterprise Wing, Singapore 138602.

Tel. +6566011679

Fax. +6566842118

e-mail: andrea@smart.mit.edu

This is the peer reviewed version of the following article A. Pavesi, M. Soncini, A. Zamperone, S. Pietronave, E. Medico, A. Redaelli, M. Prat and G. B. Fiore. Electrical conditioning of adipose-derived stem cells in a multi-chamber culture platform. Biotechnology and Bioengineering Volume 111, Issue 7, July 2014, Pages: 1452-1463 DOI: 10.1002/bit.25201, which has been published in final form at Wiley web site (http://onlinelibrary.wiley.com/doi/10.1002/bit.25201/abstract). This article may be used for non-commercial purposes in accordance with Wiley Terms and Conditions for Self-Archiving (http://olabout.wiley.com/WileyCDA/Section/id-820227.html - terms). 


\section{Abstract:}

In tissue engineering, several factors play key roles in providing adequate stimuli for cells differentiation, in particular biochemical and physical stimuli, which try to mimic the physiological microenvironments. Since electrical stimuli are important in the developing heart, we have developed an easy-to-use, costeffective cell culture platform, able to provide controlled electrical stimulation aimed at investigating the influence of the electric field in the stem cell differentiation process. This bioreactor consists of an electrical stimulator and twelve independent, petri-like culture chambers and a 3-D computational model was used to characterize the distribution and the intensity of the electric field generated in the cell culture volume. We explored the effects of monophasic and biphasic square wave pulse stimulation on a mouse adipose-derived stem cell line (m17.ASC) comparing cell viability, proliferation, protein and gene expression. Both monophasic $(8 \mathrm{~V}, 2 \mathrm{~ms}, 1 \mathrm{~Hz})$ and biphasic $(+4 \mathrm{~V}, 1 \mathrm{~ms}$ and $-4 \mathrm{~V}, 1 \mathrm{~ms} ; 1 \mathrm{~Hz})$ stimulation were compatible with cell survival and proliferation. Biphasic stimulation induced the expression of Connexin 43 , which was found to localize also at the cell membrane, which is its recognized functional mediating intercellular electrical coupling. Electrically stimulated cells showed an induced transcriptional profile more closely related to that of neonatal cadiomyocytes, particularly for biphasic stimulation. The developed platform thus allowed to set-up precise conditions to drive adult stem cells toward a myocardial phenotype solely by physical stimuli, in the absence of exogenously added expensive bioactive molecules, and can thus represent a valuable tool for translational applications for heart tissue engineering and regeneration.

Keywords: tissue engineering, electric stimulation, stimulation pattern, bioreactor, cardiac differentiation, adipose tissue derived stem cells 


\section{Introduction}

In the field of tissue engineering and regenerative medicine, where cells are cultured in vitro, it is becoming more and more evident that the microenvironment where cells grow plays a critical role, since it can instruct cells through different kinds of stimuli, which replicate the conditions experienced by cells in vivo. These stimuli can be chemical (e.g., soluble growth/differentiation factors, adhesive molecules present in the extracellular matrix, pharmacological molecules, oxygen diffusion), or physical, such as mechanical or electrical. Electrical stimuli play an important role in the field of cardiac tissue engineering and related heart cells in general. From the beginning of the heart development at the embryonic stage, the electrical stimulation is an essential component for the commitment of cardiac precursor cells. Ion currents are recognized to play a key role in cardiomyogenesis in vivo (An et al. 1996). In vitro electric stimulation can improve the functional assembly of neonatal mouse and rat cardiomyocytes into a contractile engineered cardiac tissues (Au et al. 2007; Chiu et al. 2011; Tandon et al. 2011) and promote the differentiation toward the cardiac lineage of mouse embryonal stem cells (Serena et al. 2009; Zimmermann 2011). This strategy represents a promising alternative to biochemical conditioning protocols, which were used with several cell types, including fibroblasts, bone marrow derived stem cells, human mesenchymal stem cells and embryonic stem cells, with the aim of inducing cells towards a cardiac phenotype on the basis of regulation of cardiac markers (Badorff et al. 2003; Rangappa, Entwistle, et al. 2003; Rangappa, Fen, et al. 2003). Unfortunately, most of the differentiation protocols available in literature either rely on the use of potentially dangerous drugs such as, for example, 5azacytidine (Choi et al. 2004), which being also used as anti-cancer chemotherapeutic can display related side effects or require periods of co-culture with human or mouse adult ventricular cardiomyocytes (Rangappa, Fen, et al. 2003). This clearly impairs the possibility of translation to the clinic (Miura et al. 2006). 
Endogenous electric fields are present in developing and regenerating tissues, either in the extracellular space or in the cell cytoplasm, and these fields range in strength from a few $\mathrm{mV} / \mathrm{mm}$ to several hundreds $\mathrm{mV} / \mathrm{mm}$ (Jaffe and Nuccitelli 1977; Robinson 1985). Exogenous electromagnetic fields were shown to influence in vitro the cell behavior (Zhao, Forrester, and McCaig 1999). Cells respond to an externally applied electrical stimulation with a passive and an active intracellular influx of ions, such as calcium and sodium (Trollinger, Isseroff, and Nuccitelli 2002; Djamgoz MBA et al. 2001), the relocalization of lipids and proteins (e.g., growth factor receptors) within the membrane (Zhao et al. 2002) and morphological changes (e.g., elongation, alignment) (Au et al. 2007). Protocols based on an electrical stimulation were applied using various experimental set ups and solutions for configuring the electrode, such as metal patterned electrodes (Tandon et al. 2010; Kong, Bursac, and Tung 2005), point-stimulation electrodes (Kong, Bursac, and Tung 2005; Bian and Tung 2006) or rod-shaped electrodes (Sathaye et al. 2006; Berger et al. 1994; Vunjak-Novakovic and Tandon 2009). In the case of cell monolayers, carbon rod electrodes were used to stimulate cell growth in the middle region of the applied electric field (Au et al. 2007). Different electrical stimulation protocols have been reported to improve the functional assembly of cardiomyocytes into contractile engineered tissue (Tandon et al. 2011; Tandon, Cannizzaro, et al. 2009; Barash et al. 2010). In this scenario, electrical-based bioreactor platforms are expected to enable the exploration of combinations of strictly controlled electrical stimulation with other experimental conditions that play key roles in cardiogenic differentiation (e.g., the cell developmental stage, scaffold substrates).

In this work we developed a chemical-free conditioning system based on electrical stimulation as an alternative method for inducing pre-commitment of stem cells to a cardiac phenotype. A multi-chamber bioreactor was designed to provide controlled electrical stimulation aimed at investigating the influence of the electric field in the stem cell differentiation process. The cell culture system was conceived as a user-friendly instrument with visualization and imaging compatibility and versatility in the applicable electrical stimuli. The physical phenomena and the related variables characterizing the cell culture 
environment were analyzed and quantified to optimize cell culture procedures under electrical stimulation. Computational models were used to quantify the desired stimulation in terms of electric field values and uniformity in the region where cells grow. In this way it was possible to optimize the experimental procedure in terms of the amount of culture medium necessary to guarantee favorable cell conditioning during culture. The result of the proposed design is a robust, easy-to-use, cost-effective and reproducible device able to apply different electrical stimulation protocols on cells. To validate the bioreactor tool, we explored the possibility of inducing cardiac differentiation on a mouse adiposederived stem cell line (Zamperone et al., 2013) utilizing two different electrical stimulation protocols, i.e., monophasic and biphasic stimulation, and we compared the results of these two strategies in terms of the cell viability, proliferation and global gene expression. Finally, we evaluated the levels of Cx-43 (Hahn et al. 2008; Mills et al. 2007) in order to compare control culture samples to samples stimulated electrically within the bioreactor device.

\section{Materials and Methods}

\subsection{Design and fabrication of the cell culture platform}

The general layout of the culture platform (Fig.1a) consisted of the following components: i) interchangeable silicone culture chambers, ii) two chassis capable of hosting up to six culture chambers each, and providing the cable connections to the electrical stimulator, iii) a PC-programmable electrical stimulator for the generation of pulses. All of the components were made in-house.

Culture chambers - Each culture chamber consisted of a transparent, rectangular-based culture plate $(30 \mathrm{x}$ $15 \mathrm{~mm} ; 12 \mathrm{~mm}$ in height) made using polydimethylsiloxane (PDMS, Silgard $184{ }^{\circledR}$, DownCorning), which was prepared by casting and curing within molds manufactured for this purpose. The bottom of the chamber was made flat and fully transparent by accurate surface polishing of the molds, to allow for optimal visualization of the cells using an inverted microscope. Each PDMS chamber (Fig.1b) was

provided with two parallel embedded electrodes fabricated of non-toxic (Dymond et al. 1970; Babb and 
Kupfer 1984; Merrill, Bikson, and Jefferys 2005) AISI 316 stainless steel rods (2.5 mm in diameter), placed $12 \mathrm{~mm}$ apart. Each electrode exhibited one-fourth of its side surface towards the inner volume of the chamber to ensure electrical continuity with the culture medium.

The chamber mold was manufactured using a computer numerical control (CNC) milling machine (Roland MDX-40). The electrodes were positioned inside of the mold during the mold assembly, and PDMS was then cast and cured at $80^{\circ} \mathrm{C}$ for 3 hours. Each chamber was further equipped with two additional polytetrafluoroethylene (PTFE) clamps, which were used to push the glass slide where the cells were cultured against the bottom of the chamber during each user operation (e.g., cell seeding, medium addition/removal, chambers locking/unlocking in the chassis and imaging operations).

Chassis - The chassis frame was fabricated from PMMA (Fig.1a). The locking system, which secured each chamber to the chassis was based on geometrical interference between the chamber and the chassis basement; spring gold coated connectors embedded in the back wall of the chassis allowed the chamber electrodes to connect to the stimulation apparatus. Each part of the chassis was CNC-manufactured (Modela Roland MDX-40), subsequently the parts were assembled with acrylic glue.

Electrical stimulator - An electrical stimulator able to generate monophasic and biphasic voltage stimulation with adjustable square waveforms (amplitude, pulse width and frequency) was designed and integrated in the culture system (Fig.1a). A low-cost, open source I/O board (Arduino UNO, Smart Projects, Strambino, TO, Italy) (D'Ausilio 2012) coupled with a National Instrument data acquisition board (PCMCIA, NI DaqCard6024E, National Instrument, Austin, TX) was used for this purpose. The acquisition board acquires voltage at the electrode level, allowing for signal visualization and recording. The electrical stimulator is located outside of the incubator; cables connect the stimulator output sockets (top side of the stimulator, Fig.1a) with the input sockets placed on the lateral side of each chassis.

Experimental assessment - Bench testing was performed using voltage measurements inside of the cell culture region. An electrode comb comprising six 1.27-mm-spaced gold pins was immersed in the culture medium at half the width of the chamber, along a line perpendicular to the chamber electrodes. The 
stimulator was configured at different stimulation conditions, and the time course of the chamber electrode voltage and of the voltage of each pin was simultaneously recorded (NI DAQCard-6024E, National Instruments, Austin, TX) at an acquisition rate of $25 \mathrm{kHz}$. All voltage measurements referred to one chamber electrode, which was used as the system ground.

\subsection{Finite element modelling of the electric field in the culture chambers}

A computational model was developed with the finite element method (FEM) to investigate the spatial distribution of the electric field within the culture chamber. It was assumed that the electrically stimulated cells were seeded and grown as a monolayer on a 10.5 x $22 \mathrm{~mm}$ rectangular glass substrate, which lay on the chamber bottom between the two electrodes. The overall $3 \mathrm{D}$ geometry of the cultivation system was modeled as four divided, homogeneous sub-domains (the PDMS culture chamber, electrodes, glass substrate and culture medium volume), each with their respective electrical properties.

The electric field distribution in the $3 \mathrm{D}$ environment of the culture system was simulated with Comsol multi-physics 4 (Comsol Inc., Burlington, MA, USA). Direct-current, steady state conditions were assumed, for which the governing equation solved is:

$$
-\nabla \cdot\left(\sigma \nabla V-J_{\mathrm{e}}\right)=Q_{\mathrm{i}}
$$

where $V$ is the electric potential, $\sigma$ is the electric conductivity, $J_{\mathrm{e}}$ and $Q_{\mathrm{i}}$ are an externally generated current density and the local current source, respectively (both identically set to zero in our simulations). Solving Eq. 1 yielded the distribution of the electric potential $V$ throughout the 3D domain; the electric field distribution was then derived by computing the gradient of the potential: $\mathbf{E}=-\nabla V$.

The values of $\sigma$ for the PDMS, culture medium (Tandon et al. 2011; Tandon et al. 2010), glass and stainless steel, used for the model are summarized in Table 1. The cell monolayer was not explicitly modeled but instead was considered part of the culture medium volume due to its high water content (Barash et al. 2010). The boundary conditions were prescribed as uniform potentials at the inlet or outlet faces of the chamber electrodes ( $8 \mathrm{~V}$ at the positive electrode, $0 \mathrm{~V}$ at the ground electrode). An insulated boundary condition was applied at all other external surfaces. 
A parametric analysis of the effect exerted by the volume of the culture medium used to fill the chamber was performed. Multiple simulations were run to evaluate different heights $h$ of the culture medium subdomain ( $h=2$ to $11 \mathrm{~mm}$, step $1 \mathrm{~mm}$ ), representing different volume conditions while all other conditions were unchanged.

\subsection{Cell culture experiments under electrical stimulation conditions}

Cell culture source type and preparation - The m17.ASC line was obtained from inguinal subcutaneous adipose tissue of fvb/n strain mice by limiting diluition after immuno-magnetic selection and enrichment

for Sca-1 positive cells (Zamperone et al., 2013) These cells were cultured in Claycomb medium (Sigma Aldrich) supplemented with $10 \%$ fetal bovine serum (Lonza-Cambrex), $50 \mathrm{IU} / \mathrm{ml}$ penicillin, and 100 $\mu \mathrm{g} / \mathrm{ml}$ streptomycin (Lonza-Cambrex), at $37^{\circ} \mathrm{C}$ in a humidified atmosphere of $5 \% \mathrm{CO}_{2} . \mathrm{m} 17 . \mathrm{ASC}$ were maintained in culture for more than 2 years and were passaged twice per week at subconfluency. These cells were shown to express the main mesenchymal stemness markers (Sca-1, nestin, nucleostemin, CD44, CD106; low expression of CD117; negative for CD45, CD31, and F-480) (Zamperone et al., 2013). Neonatal cardiomyocytes (nCMs) were isolated from hearts of 1-3-day-old $\mathrm{FVB} / \mathrm{N}$ mice, as indicated in the manufacturer's instructions (kit by Worthington Biochemical Corp.). Briefly as reported in our previous publication (Forte et al., 2011), immediately after isolation, cells were pre-plated for $2 \mathrm{~h}$ 30 min to recover the non-adherent-enriched fraction of $\mathrm{nCMs}$, which were then seeded on fibronectin (2 $\mathrm{mg} / \mathrm{mL}$; Sigma-Aldrich), laminin $(0.2 \%)$, gelatin $(0.02 \%)$ pre-coated plates. Adherent cells were expanded when subconfluent up to two times.

Electrical stimulation protocol - Cells were seeded on glass slides (10.5 x $22 \mathrm{~mm}$, Marienfeld, LaudaKonigshofen, Germany) and cultured in static conditions inside the PDMS culture chambers for $24 \mathrm{~h}$ followed by electrical stimulation. Two different protocols were used to electrically stimulate the adipose-derived stem cells (Berger et al. 1994; Barr and Plonsey 2004; Nuccitelli 1992; Radisic et al. 2004; Tandon, Goh, et al. 2009). Six chambers were stimulated with monophasic ( $8 \mathrm{~V}$ for $2 \mathrm{~ms}, 1 \mathrm{~Hz}$ ), and six chambers with biphasic square-wave pulses ( $+4 \mathrm{~V}$ for $1 \mathrm{~ms}$, $-4 \mathrm{~V}$ for $1 \mathrm{~ms}, 1 \mathrm{~Hz}$ ) for $72 \mathrm{~h}$. A $2 \mathrm{~ms}$ 
pulse duration was adopted as previously described in the literature (Barash et al. 2010). The stimulation signal duration was sufficient to dissipate the double-layer effect at the electrodes between subsequent pulses (Barr and Plonsey 2004). The cells were cultured at $37^{\circ} \mathrm{C}$ and with $5 \% \mathrm{CO}_{2}$, in the medium described above. For controls, chambers without electrical stimulation were cultured in parallel with the experimental groups for $72 \mathrm{~h}$.

Cell proliferation - Cells $\left(1 \times 10^{4} /\right.$ glass slide $)$ were seeded and cultured in the absence or presence of electrical stimulation, started $24 \mathrm{~h}$ after seeding, as described above. At each time point (24, 48 and 72 hours) cells were fixed with $2.5 \%$ glutaraldehyde for $20 \mathrm{~min}$ at room temperature and then stained with Crystal Violet. Stained cells were solubilized with acetic acid and the solution absorbance was read at $595 \mathrm{~nm}$ in a Microplate Reader (Model 3550, BioRad). Cell density was calculated by the conversion of absorbance to the relative cell number value by using a standard curve previously prepared. Time zero was considered the time point before electric stimulation ( 24 hours after cell seeding). Each experimental condition was assessed in triplicate in three separate experiments.

Immunofluorescence analysis - Cells were washed with PBS, fixed with 3\% paraformaldehyde (PFA) in PBS for $20 \mathrm{~min}$ at $25^{\circ} \mathrm{C}$, and permeabilized by incubation with $0.1 \%$ Triton X-100 in PBS for $1 \mathrm{~h}$. Cells were then blocked by incubation with 4\% goat serum and 1\% albumin bovine serum in PBS for 30 min and incubated with anti-connexin 43 antibody (1/400, rabbit polyclonal, ab11370, Abcam, Cambridge, UK in PBS) for $2 \mathrm{~h}$, followed by secondary Alexa Fluor ${ }^{\circledR} 488$ goat-anti-rabbit-IgG antibody (1/500, Abcam) for $45 \mathrm{~min}$ at $25^{\circ} \mathrm{C}$ in a humid chamber in the dark. Cytoskeletal actin microfilaments were stained with TRITC-phalloidin (1/100, Sigma-Aldrich and nuclei were stained with 4',6'-diamidino-2phenylindole (DAPI, 1/200, Invitrogen). m17.ASC were analyzed in 6 independent fields for chamber. Images were taken with a Leica DM5500B microscope equipped with a digital camera. Fluorescent intensity of the green channel (cx43) was evaluated with ImageJ software (Schneider et al. 2012) and then normalized for the number of cells in the range of interest. The data set was built on 10 different 
images per sample. The data where then analyzed with one way ANOVA statistical test followed by Tukey's post-hoc test.

Gene Expression Analysis - Total RNA was extracted using miRNeasy kit (QIAGEN, Hilden, Germany) and quality controlled using a Bioanalyzer 2100 (Agilent Technologies, Santa Clara, CA). Microarray analysis was performed using MOUSEWG-6_V2 Beadchips (Illumina, San Diego, CA), according to standard protocols. Raw data were processed and cubic-spline normalized using the GenomeStudio software (Illumina, San Diego, CA). Analysis for selection of differentially expressed genes and heatmap generation was carried out with Excel (Microsoft, Redmond, WA). All samples from stimulated cells were compared to control unstimulated cells to assess whether transcriptional changes induced by electrical conditioning were concordant with expression differences between neonatal cardiomyocytes and controls. Functional annotation of the genes, as well as gene pathway analysis was carried out with the online DAVID tool (see supplementary data).

\section{Results}

\subsection{Bench test assessment}

Figure 2 shows representative results from the bench test sessions involving voltage measurements within the medium-filled culture chamber, when the voltage applied to the electrodes was a biphasic square wave pulse (Fig.2a, $+4 \mathrm{~V}$ for $1 \mathrm{~ms},-4 \mathrm{~V}$ for $1 \mathrm{~ms}, 1 \mathrm{~Hz}$ pulse frequency) or a monophasic square wave pulse (Fig.2b, $+8 \mathrm{~V}$ for $2 \mathrm{~ms}, 1 \mathrm{~Hz}$ ). The time courses of the voltage, measured with respect to the ground, at the non-grounded stimulation electrode and at different positions between the electrodes are shown (Fig.2).

The temporal variation in the voltage measured at the stimulation electrode closely resembled an ideal square wave with little superimposed noise (about $1 \%$ in amplitude). The voltage time plots measured at the differently positioned pins show an initial step change that is synchronous with each edge of the stimulation signal followed by a dynamic behavior, which is related to the polarization of the culture 
medium and the shielding effect of charges cumulating at the electrode-to-medium interface. An approximate analysis of this time response yielded a value of $\sim 3 \mathrm{~ms}$ for the time constant of the system.

\subsection{FEM modeling}

The computational results allowed for a description of the $3 \mathrm{D}$ pattern of the electric field and current density throughout the computational sub-domains, particularly in the area where cells are seeded and cultured, i.e., immediately above the surface separating the culture medium from the glass substrate. Because homogeneity was assumed for the electrical properties of each material, the electric fields and current densities are proportional within each sub-domain. Representative results are shown in Fig.3 for a simulation performed with an $8 \mathrm{~V}$ voltage difference and a culture medium height of $6 \mathrm{~mm}$ in the chamber. Panels a, b and c of Figure 3 depict cross-sectional maps of the orthogonal current density components along the directions aligned with the width (x), height $(\mathrm{y})$ and depth $(\mathrm{z})$ of the chamber, respectively. The color maps show that in the culture medium region the current density in the $x$ (Fig.3a) direction is negligible relative to the current density in the $\mathrm{z}$ or $\mathrm{y}$ directions (Fig.3b, 3c), which implies that the electrode-electrode current flow is mainly in-plane on the cross section of the chamber. The corresponding cross-sectional pattern of the electric field, shown in the vector diagram in Fig.3d, represents the behavior of the field in the lower region of the culture medium sub-domain. In this region, the vectors are essentially parallel to the medium-glass interface, which ultimately means that in the region where the cell monolayer is cultured, the electric field is well aligned along the electrode-electrode direction. The current density value in the cell-culture region ranged from 600 to $800 \mathrm{~A} / \mathrm{m}^{2}$ for an $8 \mathrm{~V}$ voltage difference applied between the electrodes. Correspondingly, the electric field ranged approximately from 4.5 to $6.0 \mathrm{~V} / \mathrm{cm}$ between the electrodes, whereas the field reached values up to 9 $\mathrm{V} / \mathrm{cm}$ in the proximity of the electrode.

The results of the parametric analysis performed with different heights of the culture medium sub-domain are summarized in Fig.4. The main diagram in Fig.4 depicts the effects of medium level changes on the electric field at the cell-seeded surface. The electric field measured halfway between the electrodes 
decreases sensibly (from 5.9 to $4.2 \mathrm{~V} / \mathrm{cm} ;-29 \%$ ) when the culture medium level is increased from $2 \mathrm{~mm}$ (corresponding to an approximate medium volume of $0.9 \mathrm{ml}$ ) to $11 \mathrm{~mm}$ (approximately $5 \mathrm{ml}$ ). Concomitantly, a change occurs in the electric field behavior along the line connecting the electrodes (see insets in Fig.4, where field profiles are plotted against a silhouette of the chamber cross section). Although the field is consistently concentrated near the electrodes, flattened profiles are observed for lower medium levels, which result in a relatively uniform electric field at the cell-seeded surface (e.g., for $\mathrm{h}=2 \mathrm{~mm}$, a maximum deviation of $\sim 10 \%$ is calculated at the lateral ends of the glass slide). Conversely, for increasing levels, U-shaped profiles are formed, causing field distortion to progressively invade the region inhabited by the cell culture.

\subsection{Effects of the electric stimulation on cell behaviour}

To investigate the role of mono- and bi-phasic electric stimulation on m17.ASC proliferation, cells were treated for $72 \mathrm{~h}$ under both protocols and at each time point the cell number was measured by crystal violet staining. No significant differences were observed upon electric stimulation compared to untreated cells (control). In all cases, cells duplicated in the first $24 \mathrm{~h}$ of culture and then they slowed down proliferation in the following 2 days (Fig 5a).

Considering that electric stimulation should induce the expression of molecules associated with cardiac maturation and in particular with channels involved in the propagation of electric signals and ions, we investigated the expression of $\mathrm{Cx}-43$ in electrically stimulated cells (Tandon et al. 2011; Barash et al. 2010; Tandon et al. 2010; Kreuzberg, Willecke, and Bukauskas 2006; Yuan Ye, Sullivan, and Black 2011; You et al. 2011). Indeed after 3 days of biphasic stimulation cells expressed detectable $\mathrm{Cx}-43$, while this protein was virtually undetectable in not stimulated cells (Fig.5c). Biphasic stimulation was notably more effective than monophasic one. In biphasic stimulated cells, $\mathrm{Cx}-43$ was localized both within the cell cytoplasm in large perinuclear regions, which are indicative of the ongoing protein synthesis, and in well-defined aligned spots at the cell periphery, which is acknowledged to be its functional localization (Fig.5c, biphasic panels). In the case of monophasic stimulation only some 
cytoplasmic spots were evident (Fig.5c, monophasic panel). The cx43 fluorescent intensity quantification graph (Fig. 5b) shows a statistically significant intensity difference between the control and the biphasical stimulated sample. Whilst in case of monophasic stimulation the quantification shows an increment but not statistically significant.

Global gene expression profiles obtained with DNA microarrays revealed that the two stimulation protocols induced closely related transcriptomes $\left(\mathrm{R}^{2}=0.81\right)$ in m17.ASC cells (Fig. 6a). All samples were compared to control cells to assess whether transcriptional changes induced by electrical conditioning were concordant with expression differences between neonatal cardiomyocytes (NCM) and controls.

Some of these genes were already expressed or silenced in neonatal cardiomyocytes relative to not stimulated control cells $\left(\mathrm{R}^{2}=0.27\right)($ Fig.6b and $6 \mathrm{c})$. When the threshold was set at a 1.7 fold gain, out of 45,281 probe sequences analyzed, 42 gave signal in the electrically stimulated m17.ASC cells, and half of these also in neonatal cardiomyocytes, as shown in the heatmap (Fig.6d). When the threshold was set at a 1.7 fold loss, 66 of the probes gave no signal in stimulated cells relative to untreated control cells, among which 40 were shared with neonatal cardiomyocytes (Fig.6d). Gene expression changes induced by the treatment were found to be weak, but overall they are statistically significant given the high number of gene considered. Indeed a subset of genes were concordantly up-regulated or down-regulated in treated cells and in neonatal cardiomyocytes vs control untreated cells. Using the online DAVID tool we carried out functional annotation (see Supplementary Tables 1 and 2). Pathway analysis focused on these genes is reported in the Supplementary Tables 1 and 2.

\section{Discussion}

In this work we present the design of a bioreactor capable of delivering controlled electrical stimulations to the cells and tailored to the needs of a standard biological laboratory. The device was used to study the 
effect of the electrical stimulation on stem cells, in particular on the m17.ASC clonal cell line, which spontaneously stabilized from murine subcutaneous adipose tissue (Zamperone et al., 2013). As a validation test, two different electrical regimes were investigated. The applicability/usability of the bioreactor platform was demonstrated, the system functionality was evaluated with respect to the noisefree signal development and time response, and the differences between biological responses of the cells to monophasic or biphasic electrical stimulations were analyzed.

One of the most important design requirements was to supply a robust laboratory tool. To this purpose the distribution of the electric field within the culture chamber was thoroughly investigated using computational methods, to guarantee a precise knowledge of the electrical stimulation experienced by the cultured cells. Our FEM simulations were set-up assuming steady state. This approach was invoked in (Barash et al. 2010) who justified it under the assumption that the time constant of the electrodeelectrolyte analog electrical circuit is much larger than the pulse duration. Our bench-test measurements, however, showed that the extent of the system's time constant is of few milliseconds, which is comparable with the imposed pulse duration. This is in line with the findings reported in (Tandon et al. 2011) for stainless steel electrodes. In this condition, the steady-state assumption should be better interpreted as an electroquasistatic approximation of a dynamic electrical behavior (Tandon et al. 2011; Tandon et al. 2010). Each simulated map represents a snapshot taken just after the rising edge of a square pulse. This lacks information about the behavior with time, but adds key complementary awareness about the behavior in space. Particularly, with focus on the cell culture region, FEM modeling allowed us to characterize in detail the electric field alignment and uniformity and the way to practically avoid field distortions. The electric field was found to be relatively uniform over the cell-culture region, even if field perturbations were evidenced near the electrodes surface region. In addition, the simulations established a dependency between the culture medium volume and the electric field uniformity, thereby quantifying the culture medium volume to be used to enable the application of an electric field with the desired uniformity to the cell-populated surface. In particular, with a small amount of culture medium the electric 
field distribution can be reasonably assumed to be uniform throughout the culture region. An important indication to the user is that with such a system, the amount of culture medium that is present in each chamber should be chosen accurately and subsequently controlled during the culture.

An experimental evaluation of the time course of the applied electric signal showed that a voltage decrement inside the culture medium occurred during the stimulation interval. This decrement can be explained by considering the electrode-electrolyte interface phenomena associated with the use of any solid electrode (stainless steel in our case). When a solid electrode is immersed within culture medium, an interface is formed between the electrode and the electrolyte, and a transduction of charge carriers from electrons in the metal electrode to ions in the electrolyte takes place. During current flow the voltage gradient in the electrolyte is generally many orders of magnitude smaller than that at the interface and a layer of ions is attracted toward the surface. An electrical double layer is thus formed. When a voltage pulse between the two electrodes is applied, the current reaches its maximum value at the beginning of the pulse as the double-layer capacitances at the two electrodes begin charging and the current is predominantly capacitive. This scenario is in agreement with the behavior of the signal acquired within the culture medium, which showed that the maximum value of the voltage spike occurs near the positive stimulation electrode, and the spike decreases closer to the ground reference electrode. Accordingly, for the preliminary tests, pulsed voltage control stimulation was preferred rather than current control stimulation because it ensures a lower unrecoverable charge accumulation during the interpulse interval (Merrill, Bikson, and Jefferys 2005). However, the use of voltage control stimulation had several disadvantages: the maximum stimulation of the excitable tissue occurred only at the beginning of the pulse when the current is as a maximum, and the stimulation efficiency decreases during the pulse as the current decreases. One possible future development will concern improving the device using current control, as to place both the driven current and the injected charge under direct control, which was not feasible using voltage control (Weinman and Mahler 1964). 
The type 316L stainless steel (a cobalt-nickel-chromium-molybdenum alloy) selected for the stimulating electrodes satisfied the biocompatibility and mechanical property requirements for the application. Moreover, due to the transparent property of the PDMS, the designed chamber allowed for the clear visualization of the cultured cells and permitted sterilization by conventional means (i.e., autoclaving). Thus the bioreactor could be manufactured using readily available laboratory materials and tools and is easily duplicable and capable of being produced in small series. In addition, the bioreactor yielded positive results in terms of the usability and reliability. The preparation of the experiments was rapid compared to similar previously published methods that use modified Petri dishes (Tandon, Cannizzaro, et al. 2009). Compared to previously described bioreactors, our system offers a tightly controlled cell culture environment, whose electrical patterns were well characterized by numerical simulations. In addition, the versatility of the 12 independent culture chambers, allows testing different experimental conditions at the same time. Thanks to optical transparency and proper dimensioning of the culture chambers, it is possible to visually follow the cell cultures and to obtain images of the cells within each chamber (chamber removal from/mounting onto the chassis is quite immediate, due to its 'plug-in' concept). Moreover, it is easy to recover the cells for different kinds of analysis (e.g., immunofluorescence, microarray).

The designed apparatus/system has proven to be biocompatible in the conditions of electric stimulation we used, since cells displayed the same kinetics of proliferation as the not stimulated cells, suggesting that from this point of view the two stimulation regimens are equivalent. In view of the fact that electric stimulation was hypothesized to drive the m17.ASC cells towards a cardiomyogenic phenotype, the expression of $\mathrm{Cx}-43$ was chosen as marker of cell differentiation (Pagliari et al. 2011), although this marker is not strictly specific for this phenotype. For example, $\mathrm{Cx}-43$ can be expressed also in cells differentiating toward the osteogenic phenotype (Minkoff et al. 1994). In the case of mature cardiomyocytes, $\mathrm{Cx}-43$ expression at the gap junctions is essential for intercellular communication because single cells must be coupled to function as a pluricellular unique complex. We report that 
biphasic stimulation is more efficient than monophasic stimulation in inducing the relocalization of $\mathrm{Cx}$ 43 at the cell membrane, which is acknowledged to be its functional localization (Smyth et al., 2010). Indeed, $\mathrm{Cx}-43$ was detected as discrete spots localized at the cell membrane, in addition to perinuclear localization, its recognized synthesis localization. Although, upon the biphasic stimulation, $\mathrm{Cx}-43$ is clearly detectable at higher levels in immunofluorescence, this was not accompanied by a higher level of transcript (in the microarray analysis the Gja1 mRNA does not change upon electric stimulation). Two possible explanations can reconcile these apparently contradictory data. In the case of biphasic stimulation the mRNA or the protein are more stable. In the former case this would allow a more efficient translation in $\mathrm{Cx} 43$ protein. Also, and not necessarily in contrast, the relocalization of the protein to the cell membrane allows to reach a threshold concentration enough to be detected in immunofluorescence. Further experiments could help to elucidate this point. Tandon and colleagues analyzed the $\mathrm{Cx}-43$ expression in a model of human mesenchymal stem cells derived from adipose tissue, which were subjected to a continuous direct electric field (Tandon, Goh, et al. 2009). They found that stimulated cells expressed $\mathrm{Cx}-43$ only transiently after $2 \mathrm{~h}$ of stimulation but not after $4 \mathrm{~h}$ of stimulation. Moreover, they reported that cells aligned perpendicularly relatively to the current direction. In contrast, such a cell response could not be clearly detected in our experiments. The discrepancy between the results reported by these two laboratories can be explained by the different design of the electrical stimulation protocols and analyzed times. Tandon and colleagues used continuous direct current, which is more involved during embryogenesis, whereas we selected pulse stimulation conditions, which are preferentially involved in cardiac syncytium development and thus should replicate the electrical environment of a more mature heart tissue in a more physiological way (Tandon et al., 2009). Indeed, the biphasic wave stimulation was reported to be more effective also by another research group (Chiu et al. 2011) and, more recently, also in a study performed on human cardiac progenitor cells in our laboratory (Pietronave et al., 2013). A possible explanation for this phenomena is that the two phases act synergistically, with the first 
one acting as a conditioning sub-threshold prepulse and the second one as the excitatory pulse (Jones et al. 1987; Tung and Borderies 1992).

The intensity $(8 \mathrm{~V} / \mathrm{cm})$, the time pulse duration $(1-2 \mathrm{~ms})$, the frequency $(1 \mathrm{~Hz})$ and the total duration of stimulation $(72 \mathrm{~h})$ used in the experiments reported herein were chosen after critically reviewing the literature. Indeed they are in the physiological ranges, able to activate gene expression and, being in line with previous reports in literature $(0.1-10 \mathrm{~V} / \mathrm{cm}, 1-2 \mathrm{~ms} ; 1 \mathrm{~Hz} ; 2-4 \mathrm{~h}$ to weeks), they facilitate direct comparisons (Berger et al. 1994; Sathaye et al. 2006; Radisic et al. 2004; Au et al. 2007; Gomes et al. 2002).

As long as gene expression changes induced by the treatments are concerned, although they were found to be weak, overall they go in the direction of neonatal cardiomyocyte differentiation, with a somehow stronger effect of biphasic stimulation. Indeed, a subset of genes were concordantly up-regulated or down-regulated in treated cells and in neonatal cardiomyocytes vs untreated control cells. The positive correlations observed are statistically significant, given the high number of gene considered. In contrast to the weak effect observed here on m17.ASC cells, similar protocols applied to human cardiac progenitor cells were able to up-regulate the expression of genes typically associated with the cardiomyocytic phenotype (Pietronave et al., 2013). Such different responses to the electric stimulation probably rely on the different cell types used. Human cardiac progenitor cells are more prone to cardiac differentiation, since they were already positively influenced by the cardiac microenvironment; m17.ASC cells have not received these microenvironment-dependent instructions, and, although it is reported that adipose tissue derived stem cells can differentiate towards a cardiac phenotype (Palpant and Metzger, 2010), they probably need to receive stronger stimuli. Indeed, it is clear that the cell origin affects their future evolutive capabilities (Gaebel et al., 2011; Naftali-Shani et al., 2013). Notwithstanding the limited informations obtained in the particular case herein described, we show that exploration of gene expression changes induced by stimulation is technically feasible with this system and can be adopted for further optimization of the treatment conditions. 
Systems for studying biological electrical phenomena may be costructed to examine processes at a variety of hierarchical levels, i.e. the molecular, cellular, cell population (monolayer) and 3D tissue levels. Monolayer cultures have the advantages of requiring fewer cells, facilitating observations of changes in morphology during culture and requiring simple and well-established culture systems (e.g., tissue culture flasks and Petri dishes). However, an important drawback compared to 3D culture systems is that the monolayer culture is a less accurate representation of the in vivo conditions, in part because of a reduced extent of cell-cell communication and cell mechanics. In the future, the use of a 3D-seeded scaffold on the bottom of our culture chamber will be possible without any bioreactor modification. This is particularly important in the context of the tissue engineering. To this purpose we have herein shown that undifferentiated multipotent cells (Zamperone et al., 2013) could be induced to acquire some traits of the cardiomyocytic phenotype by electrical stimulation alone, without any additional treatment, such as the addition of biochemical factors in the culture medium. Additionally, we demonstrated a good and cost-effective method to fabricate a bioreactor tool with an easy integration potential in a standard cell culture laboratory.

\section{Acknowledgments}

We are thankful to Daniela Cantarella for technical assistance with gene expression profile. This work was supported by Cariplo Foundation (Grant 2008-2459: "Nano- micro-structured polymeric matrices for engineered cardiac prototissues") and Regione Piemonte (Ricerca sanitaria finalizzata Grant 20082981) to M.P.; S.P. was supported by a fellowship from Clinica S. Gaudenzio (Novara). 


\section{References}

An RH, Davies MP, Doevendans PA, Kubalak SW, Bangalore R, Chien KR, Kass RS. (1996). Developmental changes in beta-adrenergic modulation of L-type $\mathrm{Ca} 2+$ channels in embryonic mouse heart. Circ Res 78(3):371-378.

Asakura, Masanori, Masafumi Kitakaze, Seiji Takashima, Yulin Liao, Fuminobu Ishikura, Tsuyoshi Yoshinaka, Hiroshi Ohmoto, et al. 2002. "Cardiac Hypertrophy Is Inhibited by Antagonism of ADAM12 Processing of HB-EGF: Metalloproteinase Inhibitors as a New Therapy.." Nature Medicine 8 (1) (January): 35-40. doi:10.1038/nm0102-35.

Au, Hoi Ting H, Irene Cheng, Mohammad F Chowdhury, and Milica Radisic. 2007. "Interactive Effects of Surface Topography and Pulsatile Electrical Field Stimulation on Orientation and Elongation of Fibroblasts and Cardiomyocytes..” Biomaterials 28 (29) (October): 4277-4293. doi:10.1016/j.biomaterials.2007.06.001.

Babb, T L, and W Kupfer. 1984. "Phagocytic and Metabolic Reactions to Intracerebral Electrical Stimulation of Rat Brain..” Experimental Neurology 86 (2) (November): 183-197.

Badorff, Cornel, Ralf P Brandes, Rüdiger Popp, Stefan Rupp, Carmen Urbich, Alexandra Aicher, Ingrid Fleming, Rudi Busse, Andreas M Zeiher, and Stefanie Dimmeler. 2003. "Transdifferentiation of Blood-Derived Human Adult Endothelial Progenitor Cells Into Functionally Active Cardiomyocytes..” Circulation 107 (7) (February 25): 1024-1032.

Barash, Yiftach, Tal Dvir, Pini Tandeitnik, Emil Ruvinov, Hugo Guterman, and Smadar Cohen. 2010. “Electric Field Stimulation Integrated Into Perfusion Bioreactor for Cardiac Tissue Engineering..”

Tissue Engineering. Part C, Methods $16 \quad$ (6) (December): 1417-1426. doi:10.1089/ten.TEC.2010.0068.

Barr, Roger C, and Robert Plonsey. 2004. "Field Stimulation of 2-D Sheets of Excitable Tissue.." IEEE Transactions on Bio-Medical Engineering $51 \quad$ (3) (March): 539-540. 
doi:10.1109/TBME.2003.821042.

Bell, David, Malcolm Campbell, Matthew Ferguson, Leah Sayers, Liz Donaghy, Anna O'Regan, Victoria Jewhurst, and Mark Harbinson. 2012. “AM1-Receptor-Dependent Protection by Intermedin of Human Vascular and Cardiac Non-Vascular Cells From Ischaemia-Reperfusion Injury.." The Journal of Physiology 590 (Pt 5) (March 1): 1181-1197. doi:10.1113/jphysiol.2011.221895.

Berger, H J, S K Prasad, A J Davidoff, D Pimental, O Ellingsen, J D Marsh, T W Smith, and R A Kelly. 1994. "Continual Electric Field Stimulation Preserves Contractile Function of Adult Ventricular Myocytes in Primary Culture..” The American Journal of Physiology 266 (1 Pt 2) (January): H341-9.

Bian, Weining, and Leslie Tung. 2006. "Structure-Related Initiation of Reentry by Rapid Pacing in Monolayers of Cardiac Cells.." Circulation Research 98 (4) (March 3): e29-38. doi:10.1161/01.RES.0000209770.72203.01.

Chiu, Loraine L Y, Rohin K Iyer, John-Paul King, and Milica Radisic. 2011. "Biphasic Electrical Field Stimulation Aids in Tissue Engineering of Multicell-Type Cardiac Organoids..” Tissue Engineering Part A 17 (11-12) (June): 1465-1477. doi:10.1089/ten.tea.2007.0244.

Choi, S.C., Yoon, J., Shim, W.J., Ro, Y.M., Lim, D.S., 2004. 5-Azacytidine induces cardiac differentiation of P19 embryonic stem cells. Exp. Mol. Med. 36, 515-523

D'Ausilio, Alessandro. 2012. "Arduino: a Low-Cost Multipurpose Lab Equipment..” Behavior Research Methods 44 (2) (June): 305-313. doi:10.3758/s13428-011-0163-z.

Djamgoz MBA, M Mycielska, Z Madeja, S P Fraser, and W Korohoda. 2001. "Directional Movement of Rat Prostate Cancer Cells in Direct-Current Electric Field: Involvement of Voltagegated $\mathrm{Na}+$ Channel Activity..” Journal of Cell Science 114 (Pt 14) (July): 2697-2705.

Dymond, A M, L E Kaechele, J M Jurist, and P H Crandall. 1970. "Brain Tissue Reaction to Some Chronically Implanted Metals..” Journal of Neurosurgery 33 (5) (November): 574-580. 
doi:10.3171/jns.1970.33.5.0574.

Forte G, Pietronave S, Nardone G, Zamperone A, Magnani E, Pagliari S, Pagliari F, Giacinti C, Nicoletti C, Musaró A, Rinaldi M, Ribezzo M, Comoglio C, Traversa E, Okano T, Minieri M, Prat M, Di Nardo P. Human cardiac progenitor cell grafts as unrestricted source of supernumerary cardiac cells in healthy murine hearts. Stem Cells. 2011 Dec;29(12):2051-61.

Gaebel R, Furlani D, Sorg H, Polchow B, Frank J, Bieback K, Wang W, Klopsch C, Ong LL, Li W, Ma N, Steinhoff G. Cell origin of human mesenchymal stem cells determines a different healing performance in cardiac regeneration. PLoS One. 2011 Feb 10;6(2):e15652.

Gomes PA, de Galvao KM, Mateus EF. Excitability of isolated hearts from rats during postnatal development. J Cardiovasc Electrophysiol 2002;13:355-60.

Hahn, Joo-Yong, Hyun-Ju Cho, Hyun-Jae Kang, Tack-Seung Kim, Mi-Hyung Kim, Jung-Hwa Chung, Jang-Whan Bae, Byung-Hee Oh, Young-Bae Park, and Hyo-Soo Kim. 2008. "PreTreatment of Mesenchymal Stem Cells with a Combination of Growth Factors Enhances Gap Junction Formation, Cytoprotective Effect on Cardiomyocytes, and Therapeutic Efficacy for Myocardial Infarction.." Journal of the American College of Cardiology 51 (9) (March 4): 933943. doi:10.1016/j.jacc.2007.11.040.

Harter, C, J Pavel, F Coccia, and E Draken. 1996. "Nonclathrin Coat Protein Gamma, a Subunit of Coatomer, Binds to the Cytoplasmic Dilysine Motif of Membrane Proteins of the Early Secretory Pathway." In.

Jaffe, L F, and R Nuccitelli. 1977. "Electrical Controls of Development.." Annual Review of Biophysics and Bioengineering 6: 445-476. doi:10.1146/annurev.bb.06.060177.002305.

Jones JL, Jones RE, Balasky G. (1987). Improved cardiac cell excitation with symmetrical biphasic defibrillator waveforms. Am J Physiol 253:H1418-1424.

Kim, Jong Myung, Hyun Hwa Cho, Sun Young Lee, Chang Pyo Hong, Ji won Yang, You Sun Kim, Kuen Tak Suh, and Jin Sup Jung. 2012. "Role of IRAK1 on TNF-Induced Proliferation and NF- 
кB Activation in Human Bone Marrow Mesenchymal Stem Cells..” Cellular Physiology and Biochemistry : International Journal of Experimental Cellular Physiology, Biochemistry, and Pharmacology 30 (1): 49-60. doi:10.1159/000339045.

Kong, Chae-Ryon, Nenad Bursac, and Leslie Tung. 2005. "Mechanoelectrical Excitation by Fluid Jets in Monolayers of Cultured Cardiac Myocytes..” Journal of Applied Physiology (Bethesda, Md. : 1985) 98 (6) (June): 2328-36- discussion 2320. doi:10.1152/japplphysiol.01084.2004.

Kreuzberg, Maria M, Klaus Willecke, and Feliksas F Bukauskas. 2006. “Connexin-Mediated Cardiac Impulse Propagation: Connexin 30.2 Slows Atrioventricular Conduction in Mouse Heart..” Trends in Cardiovascular Medicine 16 (8) (November): 266-272. doi:10.1016/j.tcm.2006.05.002. Merrill, Daniel R, Marom Bikson, and John G R Jefferys. 2005. "Electrical Stimulation of Excitable Tissue: Design of Efficacious and Safe Protocols..” Journal of Neuroscience Methods 141 (2) (February 15): 171-198. doi:10.1016/j.jneumeth.2004.10.020.

Mills, William R, Niladri Mal, Matthew J Kiedrowski, Ryan Unger, Farhad Forudi, Zoran B Popovic, Marc S Penn, and Kenneth R Laurita. 2007. “Stem Cell Therapy Enhances Electrical Viability in Myocardial Infarction..” Journal of Molecular and Cellular Cardiology 42 (2) (February): 304314. doi:10.1016/j.yjmcc.2006.09.011.

Minkoff, R, V R Rundus, S B Parker, E L Hertzberg, J G Laing, and E C Beyer. 1994. “Gap Junction Proteins Exhibit Early and Specific Expression During Intramembranous Bone Formation in the Developing Chick Mandible..” Anatomy and Embryology 190 (3) (September): 231-241.

Miura, Masako, Yasuo Miura, Hesed M Padilla-Nash, Alfredo A Molinolo, Baojin Fu, Vyomesh Patel, Byoung-Moo Seo, et al. 2006. “Accumulated Chromosomal Instability in Murine Bone Marrow Mesenchymal Stem Cells Leads to Malignant Transformation..” Stem Cells (Dayton, Ohio) 24 (4) (April): 1095-1103. doi:10.1634/stemcells.2005-0403.

Naftali-Shani N, Itzhaki-Alfia A, Landa-Rouben N, Kain D, Holbova R, Adutler-Lieber S, Molotski N, Asher E, Grupper A, Millet E, Tessone A, Winkler E, Kastrup J, Feinberg MS, Zipori D, 
Pevsner-Fischer M, Raanani E, Leor J. The origin of human mesenchymal stromal cells dictates their reparative properties. J Am Heart Assoc. 2013 Sep 30;2(5):e000253.

Nishimura, Noriyuki, Thi Van Huyen Pham, Tri Budi Hartomo, Myeong Jin Lee, Daiichiro Hasegawa, Hiroki Takeda, Keiichiro Kawasaki, et al. 2011. "Rab15 Expression Correlates with Retinoic Acid-Induced Differentiation of Neuroblastoma Cells..” Oncology Reports 26 (1) (July): 145-151. doi:10.3892/or.2011.1255.

Nuccitelli, R. 1992. "Endogenous Ionic Currents and DC Electric Fields in Multicellular Animal Tissues..” Bioelectromagnetics Suppl 1: 147-157.

Pagliari, Stefania, Ana Cristina Vilela-Silva, Giancarlo Forte, Francesca Pagliari, Corrado Mandoli, Giovanni Vozzi, Stefano Pietronave, et al. 2011. "Cooperation of Biological and Mechanical Signals in Cardiac Progenitor Cell Differentiation..” Advanced Materials (Deerfield Beach, Fla.) 23 (4) (January 25): 514-518. doi:10.1002/adma.201003479.

Palpant NJ, Metzger JM. Aesthetic cardiology: adipose-derived stem cells for myocardial repair. Curr Stem Cell Res Ther. 2010 Jun;5(2):145-52. Review.

Pietronave S, Zamperone A, Oltolina F, Colangelo D, Follenzi A, Novelli E, Diena M, Pavesi A, Consolo F, Fiore GB, Soncini M, Prat M. Mono and biphasic electrical stimulation induces a precardiac differentiation in progenitor cells isolated from human heart. Stem Cells Dev. 2013 Dec 11. [Epub ahead of print] PubMed PMID: 24328510.

Radisic, Milica, Hyoungshin Park, Helen Shing, Thomas Consi, Frederick J Schoen, Robert Langer, Lisa E Freed, and Gordana Vunjak-Novakovic. 2004. "Functional Assembly of Engineered Myocardium by Electrical Stimulation of Cardiac Myocytes Cultured on Scaffolds..” Proceedings of the National Academy of Sciences of the United States of America 101 (52) (December 28): 18129-18134. doi:10.1073/pnas.0407817101.

Rangappa, Sunil, Chen Fen, Eng Hin Lee, Ariff Bongso, Eugene Kwang Wei Sim, and Eugene Kwang Sim Wei. 2003. "Transformation of Adult Mesenchymal Stem Cells Isolated From the 
Fatty Tissue Into Cardiomyocytes..” The Annals of Thoracic Surgery 75 (3) (March): 775-779.

Rangappa, Sunil, John W C Entwistle, Andrew S Wechsler, and J Yasha Kresh. 2003. "Cardiomyocyte-Mediated Contact Programs Human Mesenchymal Stem Cells to Express Cardiogenic Phenotype.." The Journal of Thoracic and Cardiovascular Surgery 126 (1) (July): $124-132$.

Ren, Jiaqiang, Ping Jin, Marianna Sabatino, Arun Balakumaran, Ji Feng, Sergei A Kuznetsov, Harvey G Klein, Pamela G Robey, and David F Stroncek. 2011. "Global Transcriptome Analysis of Human Bone Marrow Stromal Cells (BMSC) Reveals Proliferative, Mobile and Interactive Cells That Produce Abundant Extracellular Matrix Proteins, Some of Which May Affect BMSC Potency.." Cytotherapy 13 (6) (July): 661-674. doi:10.3109/14653249.2010.548379.

Robinson, K R. 1985. "The Responses of Cells to Electrical Fields: a Review..” The Journal of Cell Biology 101 (6) (December): 2023-2027.

Sathaye, Alok, Nenad Bursac, Sean Sheehy, and Leslie Tung. 2006. "Electrical Pacing Counteracts Intrinsic Shortening of Action Potential Duration of Neonatal Rat Ventricular Cells in Culture..” Journal of Molecular and Cellular Cardiology $41 \quad$ (4) (October): 633-641. doi:10.1016/j.yjmcc.2006.06.076.

Schneider, C.A., Rasband, W.S., Eliceiri, K.W. "NIH Image to ImageJ: 25 years of image analysis". Nature Methods 9, 671-675, 2012

Serena, Elena, Elisa Figallo, Nina Tandon, Christopher Cannizzaro, Sharon Gerecht, Nicola Elvassore, and Gordana Vunjak-Novakovic. 2009. "Electrical Stimulation of Human Embryonic Stem Cells: Cardiac Differentiation and the Generation of Reactive Oxygen Species..” Experimental Cell Research $315 \quad$ (20) (December 10): 3611-3619. doi:10.1016/j.yexcr.2009.08.015.

Smyth JW, Hong TT, Gao D, Vogan JM, Jensen BC, Fong TS, Simpson PC, Stainier DY, Chi NC, Shaw RM. Limited forward trafficking of connexin 43 reduces cell-cell coupling in stressed 
human and mouse myocardium. J Clin Invest. 2010 Jan;120(1):266-79.

Tandon, Nina, Christopher Cannizzaro, Pen-Hsiu Grace Chao, Robert Maidhof, Anna Marsano, Hoi

Ting Heidi Au, Milica Radisic, and Gordana Vunjak-Novakovic. 2009. "Electrical Stimulation Systems for Cardiac Tissue Engineering..” Nature Protocols 4 (2): 155-173.

Tandon, Nina, Anna Marsano, Robert Maidhof, Keiji Numata, Chrystina Montouri-Sorrentino, Christopher Cannizzaro, Joel Voldman, and Gordana Vunjak-Novakovic. 2010. "SurfacePatterned Electrode Bioreactor for Electrical Stimulation..” Lab on a Chip 10 (6) (March 21): 692-700. doi:10.1039/b917743d.

Tandon, Nina, Anna Marsano, Robert Maidhof, Leo Wan, Hyoungshin Park, and Gordana VunjakNovakovic. 2011. "Optimization of Electrical Stimulation Parameters for Cardiac Tissue Engineering.." Journal of Tissue Engineering and Regenerative Medicine 5 (6) (June): e115-25. doi:10.1002/term.377.

Tandon, Nina, Brian Goh, Anna Marsano, Pen-Hsiu Grace Chao, Chrystina Montouri-Sorrentino, Jeffrey Gimble, and Gordana Vunjak-Novakovic. 2009. "Alignment and Elongation of Human Adipose-Derived Stem Cells in Response to Direct-Current Electrical Stimulation.." Conference Proceedings : ... Annual International Conference of the IEEE Engineering in Medicine and Biology Society. IEEE Engineering in Medicine and Biology Society. Conference 2009: 65176521. doi:10.1109/IEMBS.2009.5333142.

Trollinger, Donna R, R Rivkah Isseroff, and Richard Nuccitelli. 2002. "Calcium Channel Blockers Inhibit Galvanotaxis in Human Keratinocytes..” Journal of Cellular Physiology 193 (1) (October): 1-9. doi:10.1002/jcp.10144.

Tung L, Borderies JR. (1992). Analysis of electric field stimulation of single cardiac muscle cells. Biophys J 63(2):371-386.

Vunjak-Novakovic, G, and N Tandon. 2009. "Challenges in Cardiac Tissue Engineering." Tissue Engineering .... 
Weinman, J, and J Mahler. 1964. “An Analysis of Electrical Properties of Metal Electrodes..” Medical Electronics \& Biological Engineering 2 (July): 299-310.

You, Jin-Oh, Marjan Rafat, George J C Ye, and Debra T Auguste. 2011. "Nanoengineering the Heart: Conductive Scaffolds Enhance Connexin 43 Expression..” Nano Letters 11 (9) (September 14): 3643-3648. doi:10.1021/n1201514a.

Yuan Ye, Kathy, Kelly Elizabeth Sullivan, and Lauren Deems Black. 2011. "Encapsulation of Cardiomyocytes in a Fibrin Hydrogel for Cardiac Tissue Engineering.." Journal of Visualized Experiments : JoVE (55). doi:10.3791/3251.

Zamperone A, Pietronave S, Merlin S, Colangelo D, Ranaldo G, Medico E, Di Scipio F, Berta GN, Follenzi A, Prat M. Isolation and Characterization of a Spontaneously Immortalized Multipotent Mesenchymal Cell Line Derived from Mouse Subcutaneous Adipose Tissue. Stem Cells Dev. 2013 Nov 1;22(21):2873-84.

Zhao, M, J V Forrester, and C D McCaig. 1999. “A Small, Physiological Electric Field Orients Cell Division.." Proceedings of the National Academy of Sciences of the United States of America 96 (9) (April 27): 4942-4946.

Zhao, Min, Jin Pu, John V Forrester, and Colin D McCaig. 2002. "Membrane Lipids, EGF Receptors, and Intracellular Signals Colocalize and Are Polarized in Epithelial Cells Moving Directionally in a Physiological Electric Field.." FASEB Journal : Official Publication of the Federation of American Societies for Experimental Biology 16 (8) (June): 857-859. doi:10.1096/fj.01-0811fje. Zimmermann, Wolfram-Hubertus. 2011. "Embryonic and Embryonic-Like Stem Cells in Heart Muscle Engineering.." Journal of Molecular and Cellular Cardiology 50 (2) (February): 320326. doi:10.1016/j.yjmcc.2010.10.027. 
Table:

\begin{tabular}{cc} 
Materials & trical Conductivity $(\sigma)[S / m]$ \\
\hline PDMS & $0.8310^{-12}$ \\
Culture medium & 1.5 \\
Glass & $110^{-14}$ \\
Stainless steel & $4.03210^{-6}$ \\
\hline
\end{tabular}

Table 1: Electrical properties of the culture chamber materials and fluid. 
Figures:


Figure 1: a) Cell culture platform consisting of two PMMA chassis, each of which is capable of hosting six independent culture chambers, and a custom-made electrical stimulator. The locking/unlocking system securing the chambers is observable on the basement of the chassis. The back panel of each chassis embeds the electrical connections for connecting the culture chamber electrodes to the electrical stimulator. b) Rectangular-shaped PDMS culture chamber with two embedded AISI 316 electrodes and its lid. c) Mold manufactured for producing PDMS culture chambers using a mold casting and curing technique. The mold consisted of two PMMA elements (transparent) and three POM elements (white). 


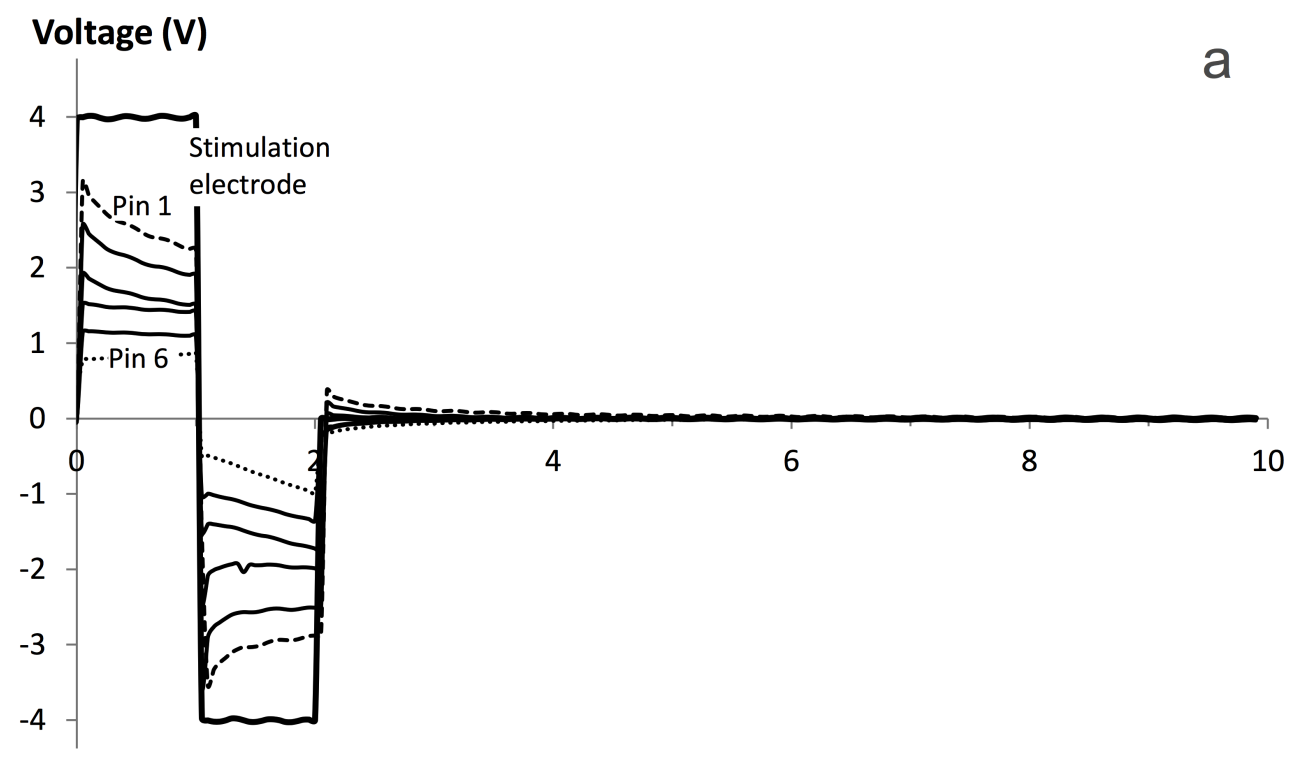

Time (ms)

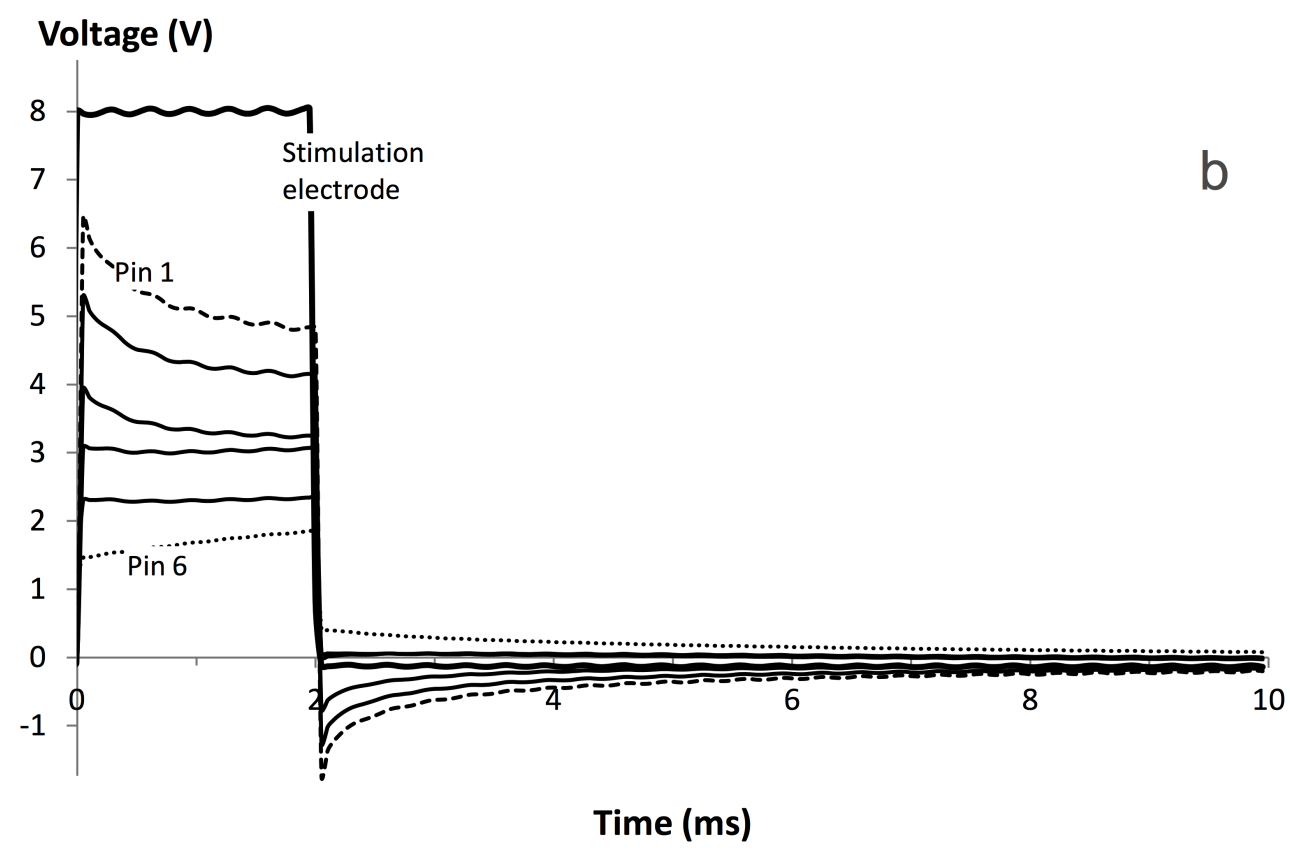

Figure 2: Plots of the voltage versus time as measured inside the medium-filled culture chamber at different positions along a line between the two stimulating electrodes: a) biphasic stimulation $(+4 \mathrm{~V}$ for $1 \mathrm{~ms},-4 \mathrm{~V}$ for $1 \mathrm{~ms}, 1 \mathrm{~Hz}$ ); b) monophasic stimulation ( $+8 \mathrm{~V}$ for $2 \mathrm{~ms}, 1 \mathrm{~Hz}$ ). The stimulation electrode channel, in blue, is connected directly with the output signal of the stimulator; the other channels are recorded values in the culture media at different distances from the stimulating electrode surface. 

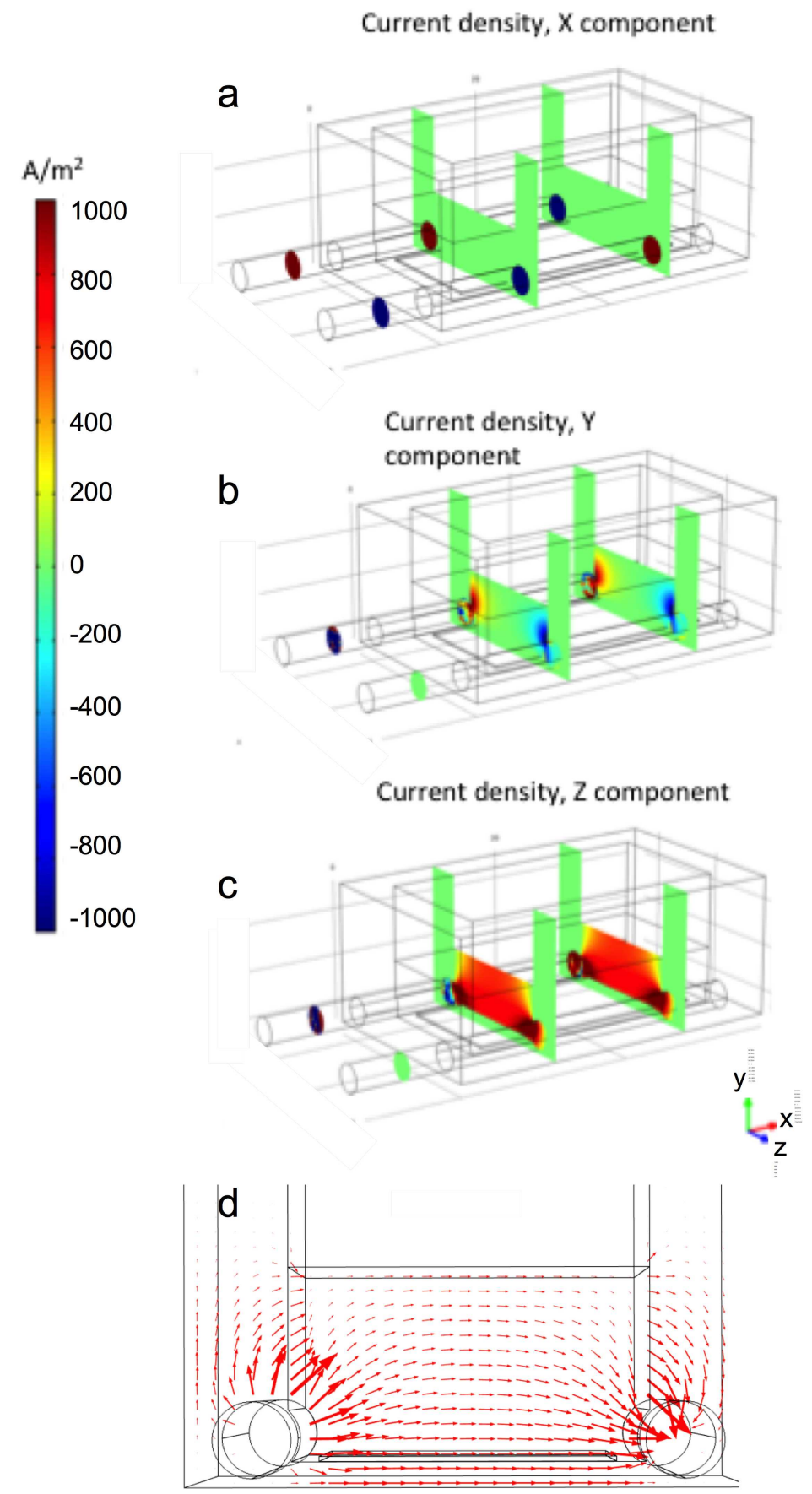

Figure 3: FEM model results. Panels a, b, c: maps of the current density components on a crosssectional plane of the culture chamber. The rectangular element on the bottom of the chamber represents the culture surface. Panel d: electric field vectors along the cross section. 


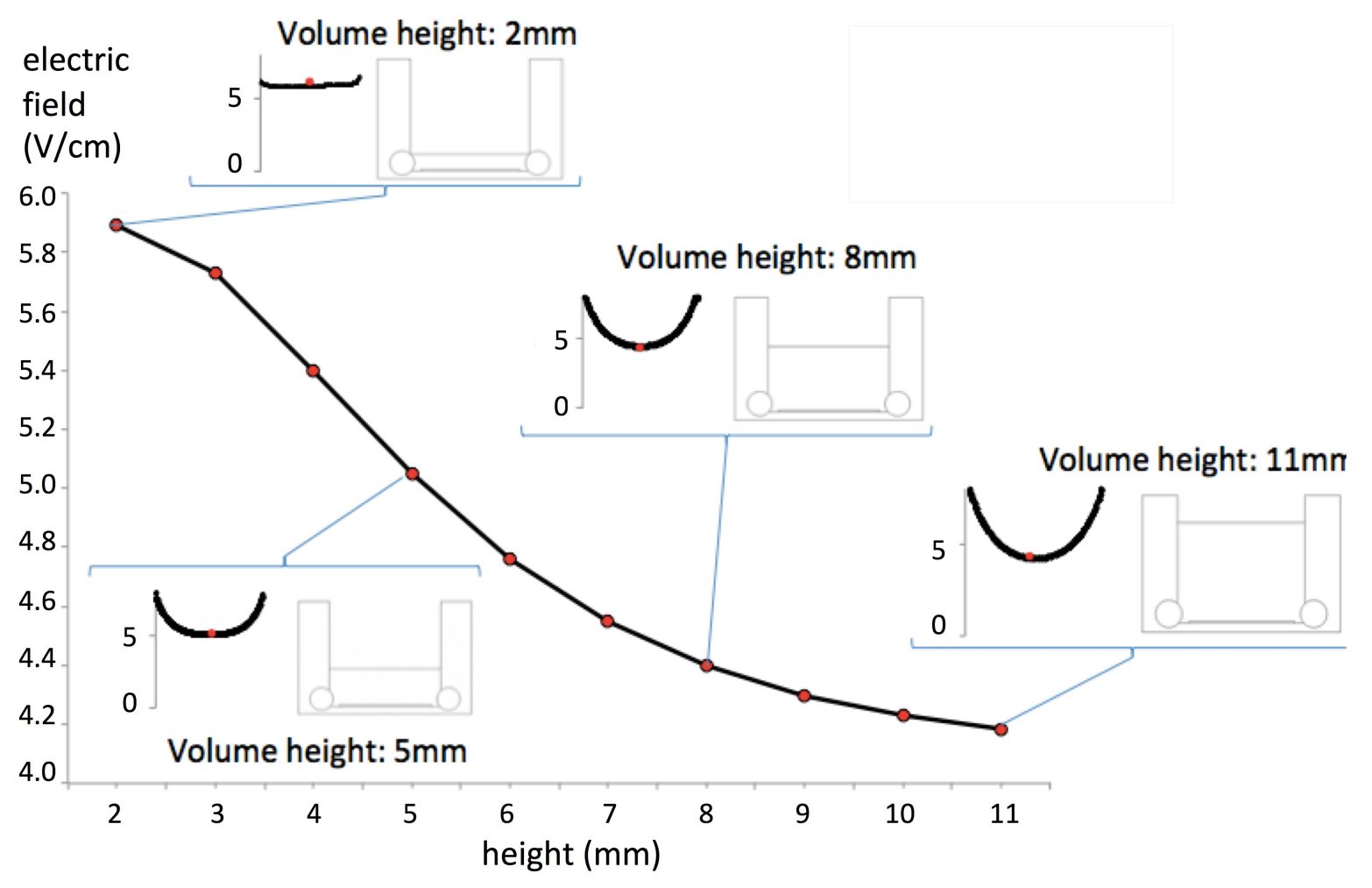

Figure 4: The behavior of the electric field $(\mathrm{V} / \mathrm{cm})$ versus changes in the culture medium height as simulated with the FEM model. The main plot shows the trend of the electric field modulus midway between the electrodes versus the height of the culture medium sub-domain. Insets show the electric field for different heights of the culture medium. In each inset, the field profile along the electrodeelectrode connecting segment is shown. 

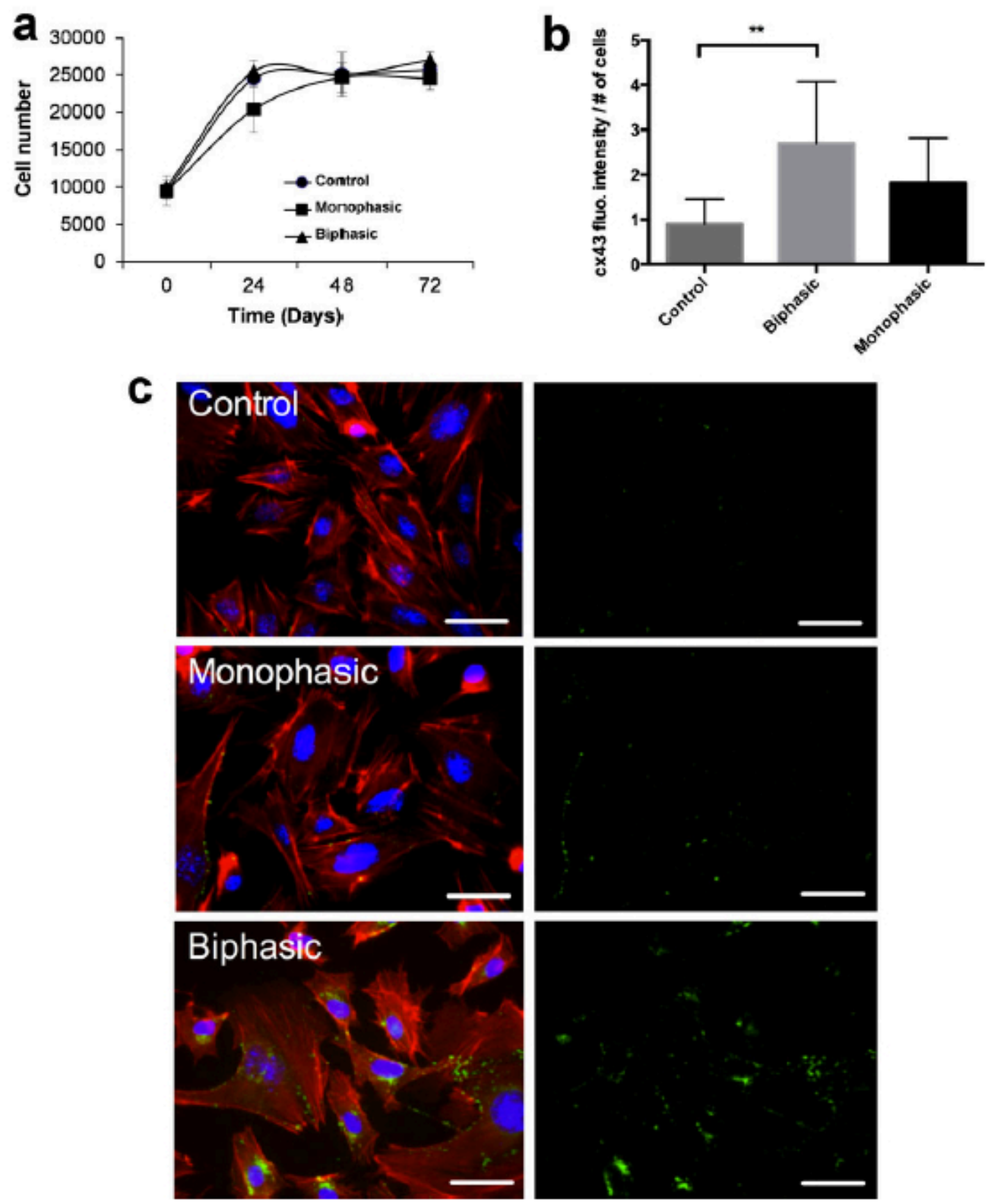

Figure 5: a) Kinetics of cell proliferation of electrically stimulated cells vs. unstimulated cells, as evaluated by Crystal Violet staining. Cell density was calculated by the conversion of absorbance to the relative cell number value by using a standard curve. No relevant differences were observed in each culture conditions ( $\mathrm{p}<0.05$, Student's test). b) Intensity fluorescent quantification graph for $\mathrm{Cx} 43$. c) Ultrastructural changes associated with the electrical stimulation of m17.ASC cells. Cells either unstimulated (control) or electrically stimulated for $72 \mathrm{~h}$ were fixed with $3 \%$ paraformaldehyde and stained with antibody against connexin-43 (green), phalloidin for actin filaments (red) and DAPI for cell nuclei (blue). Merged-channels images are shown in the left column (scale bar $75 \mathrm{~mm}$ ); single channel images of connexin-43 are shown in the right column. Positivity for connexin-43 is primarily observed when cells are stimulated with biphasic pulses and is localized at the perinuclear regions or at the plasma membrane of the cells with a dotted pattern. 

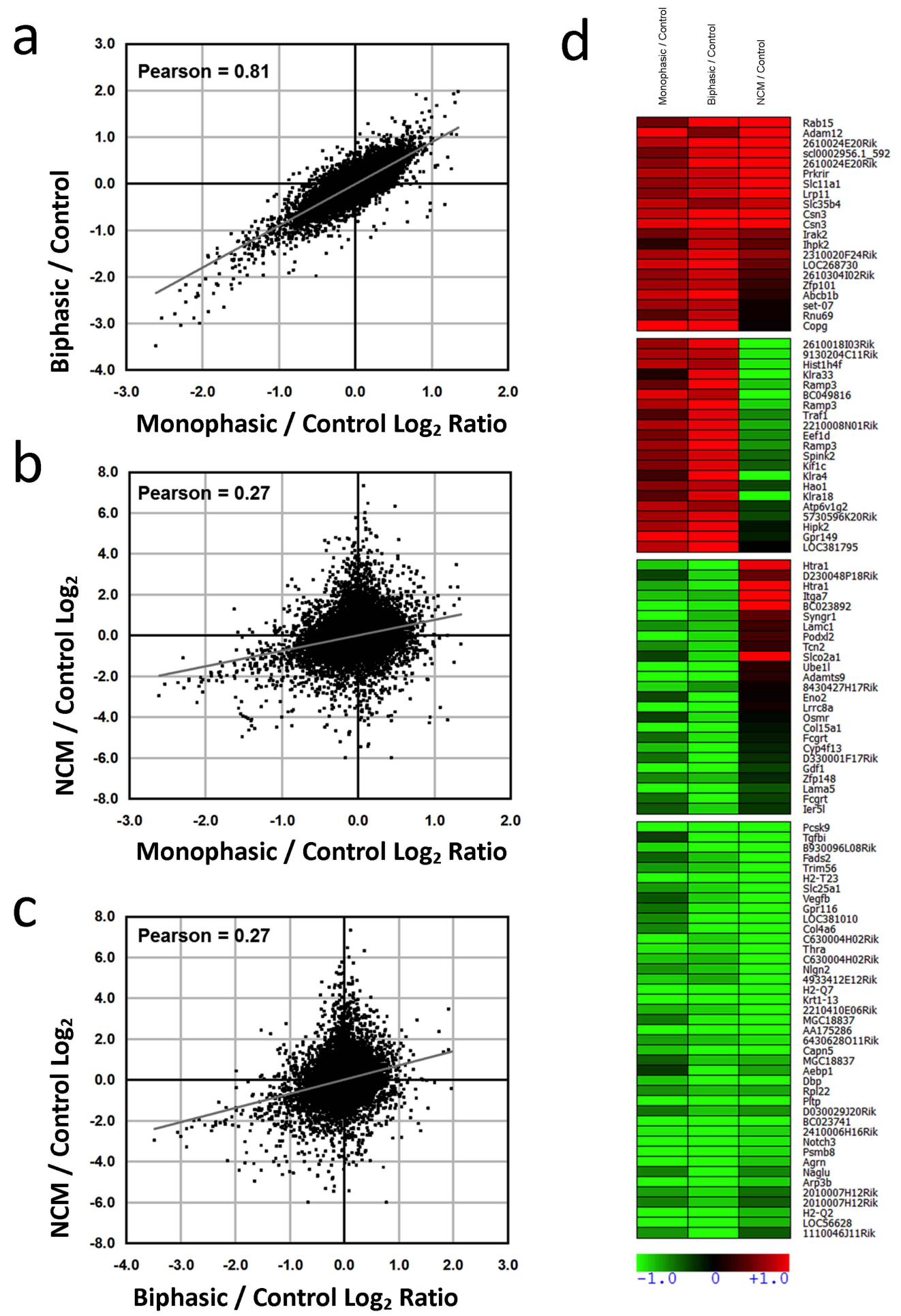

Figure 6: Global gene expression analysis; Pearson correlation values for a) changes induced by monophasic stimulation vs. changes induced by biphasic stimulation; b) changes induced by monophasic stimulation vs. changes in Neonatal Cardiomyocytes (NMC) relative to unstimulated control cells; c) changes induced by biphasic stimulation vs. changes in NMC relative to unstimulated control cells. d) Transcriptional signature of electrically stimulated cells. Significantly modulated genes reported in the heatmap are clustered in four groups according to their expression changes in stimulated cells and in NCM vs. unstimulated control cells. 